\title{
AN OBJECTIVE EFFICIENT TABU SEARCH ALGORITHM FOR TRAVELLING SALESMAN PROBLEM WITH UNCERTAIN PARAMETERS
}

\section{T. LEELAVATHY \& K. GANESAN}

Department of Mathematics, Faculty of Engineering and Technology, SRM Institute of Science and Technology,

Kattankulathur, Chennai, India

\begin{abstract}
The tabu search is a metaheuristic procedure for global optimization of travelling salesman problem. It is a dynamic neighbourhood search method. It uses flexible memory to restrict the next solution choice to some subset of neighbourhood of current solution. In this manuscript a fuzzy travelling salesman problem with trapezoidal fuzzy number is interpreted with the efficient tabu search algorithm to obtain the efficient routing plan which minimizes the length of the tour. The trapezoidal fuzzy numbers are converted into fuzzy parametric form with specified coding and then with the streamlined fuzzy arithmetic operation defined for the parametric fuzzy numbers, the tabu search iterations are carried on to get the optimal solution for the travelling salesman problem with uncertain parameters. In particular, the problem is solved without converting the fuzzy number into crisp numbers. Further, the methodology is effectively illustrated with a numerical example.

KEYWORDS: Tabu Search, Global Optimization, Vehicle Routing Problem, Trapezoidal Fuzzy Number \& Fuzzy Arithmetic Operation
\end{abstract}

Received: Jun 08, 2020; Accepted: Jun 28, 2020; Published: Sep 17, 2020; Paper Id.: IJMPERDJUN20201303

\section{INTRODUCTION}

"The most famous classic combinatorial optimization problem is known as the travelling salesman problem. It is a problem of finding a optimal route of travel with the constraint that the track should visit each point only once and return back to the starting point. The fuzzy travelling salesman" problem(FTSP) with the objective coefficient represented as trapezoidal fuzzy number is solved using evolutionary "search algorithm called tabu search algorithm. It is a dynamic neighbourhood search method which uses flexible memory to restrict the next solution. Tabu search algorithm has three main strategies such as forbidding, freeing and short-term." It controls what enters and exits the tabu list and manages "interplay between the forbidding and freeing strategies to select the trial solution. Tabu search is an improved heuristic moves from one solution to the next in search of atleast nearly optimal solution. The method of moving from one solution to another is described by a set of rules and is called a move. The set of all solutions that can be reached from a given solution using a pre-specified move is called the neighborhood of the solution. Sometime the process will cycle back to the same local optimum. A tabu list records these forbidden moves which are referred to as tabu moves. The use of memory to guide the search by using tabu list is the distinctive feature of tabu search."

The fuzzy set theory which handles the uncertainty was first implemented by Zadeh [11]. Osman [9] discussed the influence of the choice of moves on the solution. Various deviations on the travelling salesman problem include the resource constrained were discussed by Miller [7]. Clarke [3] explained the parallel and sequential version 
in which routes are merged based on maximum savings possible and expanded until no feasible route is possible. There is also a more formalized approach called the functional approach determine tabu tenures was analysed by Malek [6] and Semet[10] . Also Dubois [5] discussed in detail the theory of fuzzy sets and fuzzy numbers

In this write-up a local improvement is made to a initial solution of FTSP to obtain the global optimum using a heuristic search algorithm. Initially a trial solution is assumed and a sub-tour reversal is processed as iterations in search of a best optimal route for the fuzzy travelling salesman problem. Further the article is directed as, section 2 the preliminaries are reviewed. In section 3 the mathematical formulation of fuzzy travelling salesman problem and the related results were discussed. In section 4 the fuzzy tabu search algorithm is proposed based on the fuzzy arithmetic operations and in section 5 the methodology is explained with an example.

\section{METHODS AND MATERIALS}

Definition: "A fuzzy number $\tilde{A}$ is a trapezoidal fuzzy number denoted by $\tilde{A}=\left(a_{1}, a_{2}, a_{3}, a_{4}\right)$, where $a_{1}, a_{2}, a_{3}, a_{4}$ are real numbers and its membership function $\tilde{A}(x)$ is given below,

$$
\tilde{A}(x)=\left\{\begin{array}{cl}
\frac{x-a_{1}}{a_{2}-a_{1}}, & \text { if } a_{1} \leq x \leq a_{2} \\
1, & \text { if } a_{2} \leq x \leq a_{3} \\
\frac{a_{4}-x}{a_{4}-a_{3}}, & \text { if } a_{3} \leq x \leq a_{4} \\
0, & \text { otherwise }
\end{array}\right.
$$

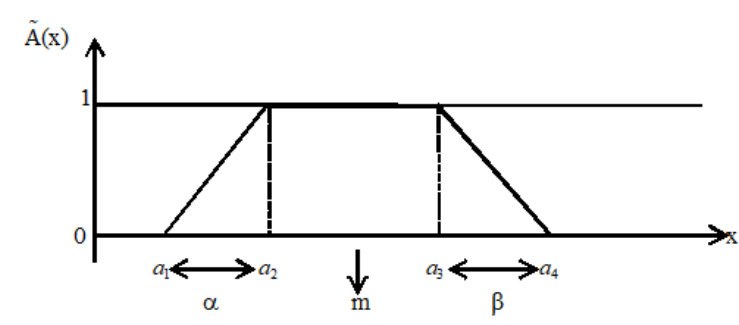

Figure 1: Trapezoidal Fuzzy Number $\tilde{\mathrm{A}}=\left(\mathrm{a}_{1}, \mathrm{a}_{2}, \mathrm{a}_{3}, \mathrm{a}_{4}\right)$.

In figure $1, \mathrm{~m}=\frac{\left(\mathrm{a}_{2}+\mathrm{a}_{3}\right)}{2}$ represents the midpoint of the core $\left[\mathrm{a}_{2}, \mathrm{a}_{3}\right], \alpha=\left(\mathrm{a}_{2}-\mathrm{a}_{1}\right)$ represents the left spread and $\beta=\left(a_{4}-a_{3}\right)$ represents the right spread of the trapezoidal fuzzy number $\tilde{A}(x)=\left(a_{1}, a_{2}, a_{3}, a_{4}\right)$.

"A trapezoidal fuzzy number $\tilde{\mathrm{A}} \in \mathrm{F}(\mathrm{R})$ can also be represented as a pair $\tilde{\mathrm{A}}=(\underline{\mathrm{a}}, \overline{\mathrm{a}})$ of functions $\underline{\mathrm{a}}(\mathrm{r})$ and $\overline{\mathrm{a}}(\mathrm{r})$ for $0 \leq \mathrm{r} \leq 1$ which satisfies the following requirements, Ming Ma [14] :

- $\underline{a}(r)$ is a bounded monotonic increasing left continuous function.

- $\quad \bar{a}(r)$ is a bounded monotonic decreasing left continuous function.

- $\quad \underline{\mathrm{a}}(\mathrm{r}) \leq \overline{\mathrm{a}}(\mathrm{r}), \quad 0 \leq \mathrm{r} \leq 1 . "$ 
For an arbitrary "trapezoidal fuzzy number $\tilde{\mathrm{A}}=(\underline{\mathrm{a}}, \overline{\mathrm{a}})$, the number $\mathrm{a}_{0}=\left(\frac{\underline{\mathrm{a}}(1)+\overline{\mathrm{a}}(1)}{2}\right)$ is said to be a location index number of $\tilde{\mathrm{A}}$. The two non-decreasing left continuous functions, $\mathrm{a}_{*}=\left(\mathrm{a}_{0}-\underline{\mathrm{a}}\right), \mathrm{a}^{*}=\left(\overline{\mathrm{a}}-\mathrm{a}_{0}\right)$ are called the left fuzziness index function and the right fuzziness index function respectively. Hence every trapezoidal fuzzy number $\tilde{A}=\left(a_{1}, a_{2}, a_{3}, a_{4}\right)$ can also be represented by $\tilde{\mathrm{A}}=\left(\mathrm{a}_{0}, \mathrm{a}_{*}, \mathrm{a}^{*}\right) . ”$

"An efficient approach for comparing the fuzzy numbers is by the use of a ranking function based on their graded means. That is, for every $\tilde{\mathrm{A}}=\left(\mathrm{a}_{1}, \mathrm{a}_{2}, \mathrm{a}_{3}, \mathrm{a}_{4}\right) \in \mathrm{F}(\mathrm{R})$, the ranking function $\mathfrak{R}: \mathrm{F}(\mathrm{R}) \rightarrow \mathrm{R}$ by graded mean is defined as $\mathfrak{R}(\tilde{\mathrm{A}})=\left(\frac{\mathrm{a}_{*}+4 \mathrm{a}_{0}+\mathrm{a}^{*}}{6}\right)$.

\section{Arithmetic Operations on Trapezoidal Fuzzy Numbers}

For "arbitrary trapezoidal fuzzy numbers $\tilde{\mathrm{A}}=\left(\mathrm{a}_{0}, \mathrm{a}_{*}, \mathrm{a}^{*}\right)$ and $\tilde{\mathrm{B}}=\left(\mathrm{b}_{0}, \mathrm{~b}_{*}, \mathrm{~b}^{*}\right)$ and $*=\{+,-, \times, \div\}$, the arithmetic operations on the trapezoidal fuzzy numbers based upon both location index and fuzziness index functions are defined by

$$
\begin{aligned}
\tilde{\mathrm{A}} * \tilde{\mathrm{B}} & =\left(\mathrm{a}_{0} * \mathrm{~b}_{0}, \mathrm{a}_{*} \vee \mathrm{b}_{*}, \mathrm{a}^{*} \vee \mathrm{b}^{*}\right) \\
& =\left(\mathrm{a}_{0} * \mathrm{~b}_{0}, \max \left\{\mathrm{a}_{*}, \mathrm{~b}_{*}\right\}, \max \left\{\mathrm{a}^{*}, \mathrm{~b}^{*}\right\}\right) .
\end{aligned}
$$

In particular for any two triangular fuzzy "numbers $\tilde{\mathrm{A}}=\left(\mathrm{a}_{0}, \mathrm{a}_{*}, \mathrm{a}^{*}\right)$ and $\tilde{\mathrm{B}}=\left(\mathrm{b}_{0}, \mathrm{~b}_{*}, \mathrm{~b}^{*}\right)$, we define:

(i) Addition: $\tilde{\mathrm{A}}+\tilde{\mathrm{B}}=\left(\mathrm{a}_{0}, \mathrm{a}_{*}, \mathrm{a}^{*}\right)+\left(\mathrm{b}_{0}, \mathrm{~b}_{*}, \mathrm{~b}^{*}\right)=\left(\mathrm{a}_{0}+\mathrm{b}_{0}, \mathrm{a}_{*} \vee \mathrm{b}_{*}, \mathrm{a}^{*} \vee \mathrm{b}^{*}\right)$

$$
=\left(a_{0}+b_{0}, \max \left\{a_{*}, b_{*}\right\}, \max \left\{a^{*}, b^{*}\right\}\right)
$$

(ii) Subtraction: $\tilde{\mathrm{A}}-\tilde{\mathrm{B}}=\left(\mathrm{a}_{0}, \mathrm{a}_{*}, \mathrm{a}^{*}\right)-\left(\mathrm{b}_{0}, \mathrm{~b}_{*}, \mathrm{~b}^{*}\right)$

$$
=\left(a_{0}-b_{0}, \max \left\{a_{*}, b_{*}\right\}, \max \left\{a^{*}, b^{*}\right\}\right)
$$

(iii) Multiplication: $\tilde{\mathrm{A}} \times \tilde{\mathrm{B}}=\left(\mathrm{a}_{0}, \mathrm{a}_{*}, \mathrm{a}^{*}\right) \times\left(\mathrm{b}_{0}, \mathrm{~b}_{*}, \mathrm{~b}^{*}\right)$

$$
=\left(a_{0} \times b_{0}, \max \left\{a_{*}, b_{*}\right\}, \max \left\{a^{*}, b^{*}\right\}\right)
$$

(iv) Division: $\quad \tilde{\mathrm{A}} \div \tilde{\mathrm{B}}=\left(\mathrm{a}_{0}, \mathrm{a}_{*}, \mathrm{a}^{*}\right) \div\left(\mathrm{b}_{0}, \mathrm{~b}_{*}, \mathrm{~b}^{*}\right)$

$$
=\left(a_{0} \div b_{0}, \max \left\{a_{*}, b_{*}\right\}, \max \left\{a^{*}, b^{*}\right\}\right), \text { provided } b_{0} \neq 0
$$

(v) Scalar Multiplication: $\lambda \tilde{\mathrm{A}}=\lambda\left(\mathrm{a}_{0}, \mathrm{a}_{*}, \mathrm{a}^{*}\right)=\left(\lambda \mathrm{a}_{0}, \mathrm{a}_{*}, \mathrm{a}^{*}\right), 0 \leq \lambda \leq 1$ “

\section{FUZZY TRAVELLING SALESMAN PROBLEM}

\section{Mathematical Formulation of Fuzzy Travelling Salesman Problem}


The fuzzy travelling salesman problem aims to find the order or sequence that the salesman should visit each city, so that the total distance travelled or cost or time of travelling is minimum, with the constraint that the salesman should visit each city once and return to the starting point.

The Mathematical Formulation of Fuzzy Travelling Salesman Problem is,

Minimize $: \quad \tilde{Z} \approx \sum_{i=1}^{n} \sum_{j=1}^{n} \tilde{d}_{i j} \tilde{x}_{i j}$

subject to $\quad \sum_{i=1}^{n} \tilde{x}_{i j} \approx \tilde{1} \quad(j=1,2, \ldots \ldots \ldots . . n)$

$$
\sum_{j=1}^{n} \tilde{x}_{i j} \approx \tilde{1} \quad(i=1,2, \ldots \ldots \ldots . . n)
$$

and $\tilde{\mathrm{x}}_{i j} \approx \tilde{0}$ or $\tilde{1} \quad$ for all $i, j=1,2, \ldots \ldots \ldots . . . n$.

Also $\tilde{\mathrm{d}}_{\mathrm{ii}}$ are defined to be large positive number to ensure that all $\tilde{\mathrm{x}}_{\mathrm{ii}}(\mathrm{i}=1,2, \ldots \ldots \ldots . \mathrm{n})$ are zero.

\section{RESULTS AND DISCUSSIONS}

Flow of Fuzzy Tabu Search Algorithm

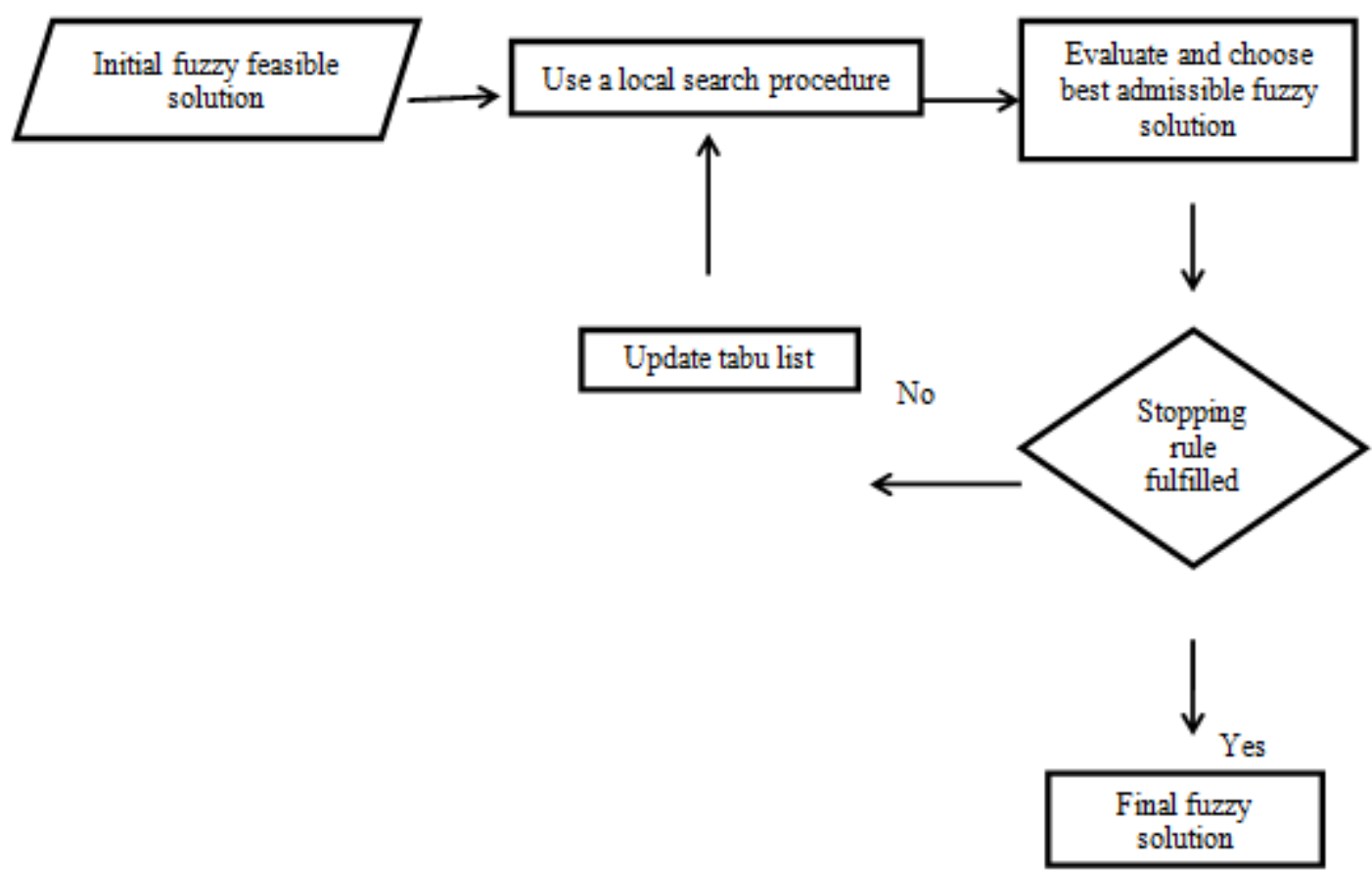

Figure-2

\section{Proposed Algorithm for Fuzzy Travelling Salesman Problem}

Initialization: Start with a fuzzy feasible initial solution $i \in S$. Set $i^{*}=i$ and $k=0$.

Iteration:

- Use an appropriate local search procedure to define the fuzzy feasible moves into a local neighborhood of the certain fuzzy trial solution. i.e., set $\mathrm{k}=\mathrm{k}+1$ and generate a subset $\mathrm{v}^{*}$ of solution in $\mathrm{N}(\mathrm{i}, \mathrm{k})$, where $\mathrm{N}$ is the neighboring 
solution.

- Avoid choosing any move on the current tabu list unless that move would result in a better fuzzy trial solution so far. i.e., choose a best $\mathrm{j}$ in $\mathrm{v}^{*}$ and set $\mathrm{i}=\mathrm{j}$. If $\mathrm{z}(\mathrm{i})<\mathrm{z}(\mathrm{j})$ then $\operatorname{set} \mathrm{i}^{*}=\mathrm{i}$.

- Adopt this solution as the next fuzzy trial solution and update the tabu list to forbid cycling back. Maximum size of tabu list should be four links, if it is more than four delete the oldest links.

- Stop after three consecutive iterations without an improvement in the best objective function value, else go to step 2.

\section{Illustration}

Consider a fuzzy travelling salesman problem of five cities with cost of travel as trapezoidal fuzzy number.

Table 1: Fuzzy Travelling Salesman Problem with Trapezoidal Fuzzy Number $\tilde{A}=\left(a_{1}, a_{2}, a_{3}, a_{4}\right)$.

\begin{tabular}{|c|c|c|c|c|}
\hline- & $(5,6,8,9)$ & $(3,4,6,7)$ & $(0,2,4,6)$ & $(3,4,6,7)$ \\
\hline$(5,6,8,9)$ & - & $(5,6,10,11)$ & $(2,3,5,6)$ & $(0,2,4,6)$ \\
\hline$(3,4,6,7)$ & $(5,6,10,11)$ & - & $(4,5,7,8)$ & $(0,1,3,4)$ \\
\hline$(0,2,4,6)$ & $(2,3,5,6)$ & $(4,5,7,8)$ & - & $(0,1,3,4)$ \\
\hline$(3,4,6,7)$ & $(0,2,4,6)$ & $(0,1,3,4)$ & $(0,1,3,4)$ & - \\
\hline
\end{tabular}

Table 2: Trapezoidal Fuzzy Number Represented in Parametric form $\tilde{A}=\left(a_{0}, a_{*}, a^{*}\right)$.

\begin{tabular}{|l|l|l|l|l|}
\hline- & $(7,2-\alpha, 2-\alpha)$ & $(5,2-\alpha, 2-\alpha)$ & $(3,3-2 \alpha, 3-2 \alpha)$ & $(5,2-\alpha, 2-\alpha)$ \\
\hline$(7,2-\alpha, 2-\alpha)$ & - & $(8,3-\alpha, 3-\alpha)$ & $(4,2-\alpha, 2-\alpha)$ & $(3,3-2 \alpha, 3-2 \alpha)$ \\
\hline$(5,2-\alpha, 2-\alpha)$ & $(8,3-\alpha, 3-\alpha)$ & - & $(6,2-\alpha, 2-\alpha)$ & $(2,2-\alpha, 2-\alpha)$ \\
\hline$(3,3-2 \alpha, 3-2 \alpha)$ & $(4,2-\alpha, 2-\alpha)$ & $(6,2-\alpha, 2-\alpha)$ & - & $(2,2-\alpha, 2-\alpha)$ \\
\hline$(5,2-\alpha, 2-\alpha)$ & $(3,3-2 \alpha, 3-2 \alpha)$ & $(2,2-\alpha, 2-\alpha)$ & $(2,2-\alpha, 2-\alpha)$ & - \\
\hline
\end{tabular}

Initial trial solution is $1-2-3-4-5-1$ and the cost is $(28,3-\alpha, 3-\alpha)$

Table 3: Iterations of Tabu Search Algorithm

\begin{tabular}{|c|c|c|c|}
\hline Iterations & 1 & 2 & 3 \\
\hline Sub tour reversal & $4-5$ & $3-5-4$ & $2-4$ \\
\hline Deleted Link & $3-4,5-1$ & $2-3,4-1$ & $1-2,4-5$ \\
\hline Added Link & $3-5,4-1$ & $2-4,3-1$ & $2-5,4-1$ \\
\hline Tabu list & $3-5,4-1$ & $3-5,2-4,3-1$ & $2-4,3-1,2-5,4-1$ \\
\hline New trial solution & $1-2-3-5-4-1$ & $1-2-4-5-3-1$ & $1-4-2-5-3-1$ \\
\hline \multicolumn{4}{|l|}{ Cost } \\
\hline Iterations & 4 & 5 & 6 \\
\hline Sub tour reversal & $2-5$ & $2-3$ & $3-2$ \\
\hline Deleted Link & $4-2,5-3$ & $5-2,3-1$ & $5-3,2-5$ \\
\hline Added Link & $4-5,2-3$ & $5-3,2-1$ & $5-2,3-1$ \\
\hline
\end{tabular}




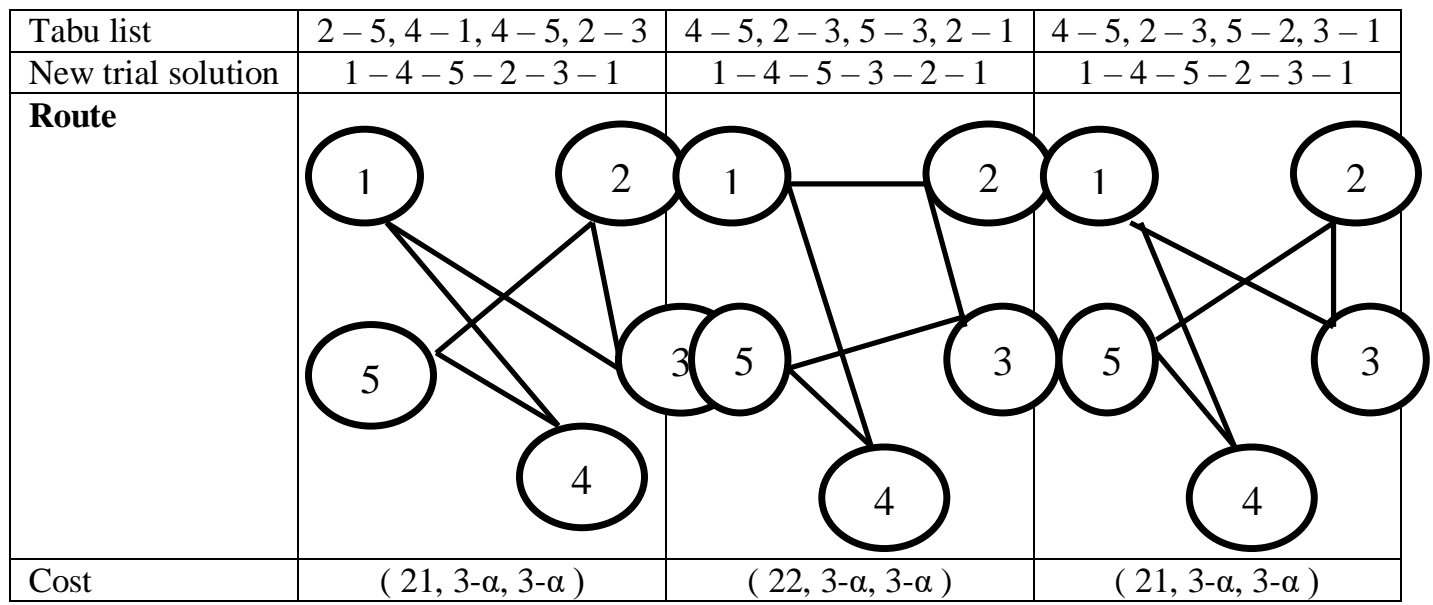

Iteration 6 should be prevented since it is cycling back to the preceding trial solution, since no other immediate neighbors are available, the stopping rule terminates the algorithm.

\section{Comparative Result}

The efficient route plan for the fuzzy travelling salesman problem with objective coefficient as trapezoidal fuzzy numbers is obtained by applying the tabu search algorithm. The best route of travel is $1-4-2-5-3-1$ with minimum fuzzy cost as (17, 3-2 $\alpha, 3-2 \alpha), \alpha \in[0,1]$. When $\alpha=0$ the minimum fuzzy cost is $(14,16,18,20)$. In particular, we obtained fuzzy optimal solution with reduced vagueness without converting the problem into classical form, which retained the physical nature of the fuzzy travelling salesman problem. For the same problem Jadunath et. al.,[6] obtained the result by converting the fuzzy numbers in to crisp numbers with approximate cost of travel as 19 which does not reflect the fuzziness of the given problem. For comparision and to portray the efficacy of the methodology, the minimum fuzzy cost is converted in to a crisp number by using the ranking function and we get it as 12.33 which is lesser than the result shown by the author [6].

\section{CONCLUSIONS}

The Uncertain optimization problems are sufficiently complex, so heuristic methods are commonly used to search for a good feasible solution atleast nearer to optimal solution. A tabu search algorithm is used to find an efficient route for the fuzzy travelling salesman problem with cost coefficients as fuzzy numbers. Tabu search moves from the current trial solution to the best neighboring trial solution at each iteration. Also it has a tabu list memory to record the forbidden moves leads to cyclic back to encourage moving towards the new parts of the feasible region. In this way we obtained the fuzzy optimal solution of the fuzzy travelling salesman problem without modifying the nature of fuzziness of the given problem.

\section{REFERENCES}

1. Bellman, R.E. and L.A. Zadeh (1970), Decision making in fuzzy environment, Management science, 17, 144-164.

2. Christofides, N. (1985). Vehicle Routing, in The Traveling Salesman Problem, (pp. 431-448), Lawler, Lenstra, RinooyKan and Shmoys, eds., John Wiley.

3. Clarke, G. and Wright, J. (1964), Scheduling of Vehicles from a Central Depot to a Number of Delivery Points, Opera-tions Research, Vol. 12,, pp. 568-581. doi:10.1287/opre.12.4.568

4. Dantzig GB, Fulkerson DR, Johnson SM (1954). Solution of a Large-scale Traveling Salesman Problem. Operations Research, 2, 393410 . 
5. Dubois, D.; Prade, H. 1987. The mean value of a fuzzy number, Fuzzy Sets and Systems 24(3): 279-300.

6. Jadunath Nayak, Sudarsan Nanda \&SrikumarAcharya (2017), Hungarian Method to Solve Travelling Salesman Problem with Fuzzy Cost, International Journal of Mathematics Trends and Technology, 49(5).

7. Malek, M., Guruswamy, M., Pandya, M. and Owens, H. (1989), Serial and Parallel Simulated Annealing and Tabu Search Algorithms for the Traveling Salesman Problem, Annals of Operations Research, Vol. 21, No. 1, pp. 59-84. doi:10.1007/BF02022093.

8. Miller and J. Pekny (1991). Exact Solution of Large Asymmetric Traveling Salesman Problems, Science 251,754-761.

9. Ming Ma, Menahem Friedman, Abraham kandel (1999), A new fuzzy arithmetic, Fuzzy sets and systems, 108,83-90.

10. Osman (1993), Metastrategy Simulated Annealing and Tabu Search Algorithms for the Vehicle Routing Problem, Annals of Operations Research, Vol. 41, pp. 421-451. doi:10.1007/BF02023004.

11. Semet, F. and Taillard, E. (1993), Solving Real-Life Vehicle Routing Problems Efficiently Using Taboo Search, Annals of Operations Research, Vol. 41, pp. 469-488. doi:10.1007/BF02023006.

12. Zadeh. L.A. (1965). Fuzzy sets, Information and Control, 8, 338-353.

13. Zimmermann, H.-J. (1978). Fuzzy programming and linear programming with several objective functions, Fuzzy Sets and Systems 1(1): 45-55. 
